\title{
The Vertex-Neighbor-Integrity of Digraphs
}

\author{
Jiangyan Guo and Elkin Vumar* \\ College of Mathematics and System Sciences, Xinjiang University, \\ Urumqi 830046, P.R.China \\ vumar@xju.edu.cn
}

\begin{abstract}
In this paper, as an extension of the concept of vertexneighbor-integrity of graphs, we introduce the notion of vertex-neighborintegrity of digraphs. Let $D=(V, A)$ be a digraph. The open and closed out-neighborhoods of a set $S \subseteq V$ are denoted by $N^{+}(S)=$ $\{v: u v \in A(D), u \in S, v \in V \backslash S\}$ and $N^{+}[S]=N^{+}(S) \cup S$, respectively. The vertex-neighbor-integrity of the digraph $D$ is defined as $V N I(D)=\min _{S \subseteq V}\left\{|S|+m\left(D / S^{+}\right)\right\}$, where $D / S^{+}:=D-N^{+}[S]$ and $m\left(D / S^{+}\right)$denotes the order of a maximum strong component of $D / S^{+}$. We first discuss some basic properties of the vertex-neighbor-integrity of digraphs, and then using these properties we study the maximum vertexneighbor-integrity which can be obtained by orienting the edges of $K_{n}$ and $K_{s, t}$.
\end{abstract}

Keywords: Vertex-neighbor-integrity, directed vertex-neighbor-integrity, orientation.

\section{Introduction}

Among several graph parameters, is the integrity introduced by Barefoot, Entringer, and Swart [1, [2] as an alternative measure of the vulnerability of graphs to disruption caused by the removal of vertices. In 1996, modeling a spy network by a graph whose vertices represent the agents and whose edges represent the lines of communication, Cozzens and $\mathrm{Wu}$ [3, 4] introduced a new graph parameter called "vertex-neighbor-integrity". In a spy network, if a spy is betrayed or arrested, then the espionage agency can no longer trust any of the spies with whom he or she was in direct communication. Clearly this means that the vertex representing the discovered spy and all its neighbors become efficiently useless to the network as a whole. Hence one way of measuring the robustness of such spy network is to consider the effect of removing a set of vertices and all of its neighbors from the graph.

Let $G=(V, E)$ be a graph and $m(G)$ be the order of a maximum component of $G$. The integrity $I(G)$ and the edge-integrity $I^{\prime}(G)$ are defined respectively as $I(G)=\min _{S \subseteq V}\{|S|+m(G-S)\}$ and $I^{\prime}(G)=\min _{T \subseteq E}\{|T|+m(G-T)\}$. A vertex subversion strategy $S$ of $G$ is a set of vertices in $G$ whose closed neighborhood

\footnotetext{
^ Corresponding author. The project sponsored by SRF for ROCS, REM.
} 
is deleted from $G$. The survival graph is denoted by $G / S$. The vertex-neighborintegrity of $G, V N I(G)$, is defined as

$$
V N I(G)=\min _{S \subseteq V}\{|S|+m(G / S)\} .
$$

For the results on the vertex-neighbor-integrity of graphs see [3], 4], 5] and [8].

Until quite recently, the studies of the vulnerability parameters of networks have focus on undirected graphs. Since many situations can be modeled by directed graphs, it is quite natural to consider such vulnerability parameters for digraphs as well. Based on this idea, Goddard and Vandell [9] extended the concept of integrity to digraphs and Vandell [10] extended edge-integrity to digraphs. They introduced in [9] and [10] respectively the integrity and the arc-integrity of digraphs. The integrity and the arc-integrity of a digraph $D=(V, A)$ are defined respectively as $I(D)=\min _{S \subseteq V}\{|S|+m(D-S)\}$ and $I^{\prime}(D)=\min _{T \subseteq A}\{|T|+m(D-T)\}$, where $m(H)$ stands for the order of a maximum strong component of $H$. In [9] and [10], after some basic properties of $I(D)$ and $I^{\prime}(D)$, the authors studied the maximum (arc-)integrity which can be obtained by orienting the edges of graphs.

Motivated by the above work of Goddard and Vandell, we extend the concept of vertex-neighbor-integrity to digraphs and introduce the directed vertexneighbor-integrity of graphs. The idea behind our extension is the following. Consider a spy network modeled by a simple digraph $D=(V, A)$ whose vertices represent agents and whose arcs represent directed line of communications, where an arc $u v$ means that $u$ is a superior of $v$, and in operations $v$ receives orders from his or her superior $u$, but never knows who $u$ is. Hence when the agent $u$ is discovered, all the subordinates of $u$ can no longer be trusted, but the superiors of $u$ are still safe for the whole network. Such a betrayals are clearly equivalent to the removal of the closed out-neighborhood of $u$ in the modeling digraph.

Let $D=(V, A)$ be a simple digraph and $u \in V(D)$. The open and closed out-neighborhoods of $u$ are defined as $N^{+}(u)=\{v \in V(D): u v \in A(D)\}$ and $N^{+}[u]=N^{+}(u) \cup\{u\}$, respectively. For a subset $S$ of $V$, the open and closed outneighborhoods are defined as $N^{+}(S)=\bigcup_{v \in S} N^{+}(v)-S$ and $N^{+}[S]=N^{+}(S) \cup S$, respectively. The open and closed in-neighborhoods of a vertex $v$ and a set $S \subseteq V$ are defined similarly. Let $d^{+}(v)=\left|N^{+}(v)\right|$ and $d^{-}(v)=\left|N^{-}(v)\right|$. We say that a vertex $u$ dominates $v$ if $u v \in A(D)$. A set $S \subseteq V$ is called a dominating set if $N^{+}[S]=V$. In particular, when $S=\{u\}$ is a dominating set we call $u$ a dominating vertex.

Following Cozzens and $\mathrm{Wu}$, we introduce the concept of vertex subversion strategy in digraphs. A vertex $u$ is said to be subverted from $D$, if the closed out-neighborhood $N^{+}[u]$ is deleted from $D$. A set of vertices $S$ in $D$ is called a vertex subversion strategy of $D$, if the closed out-neighborhood of $S$ is deleted from $D$, i.e., each of the vertices in $S$ has been subverted from $D$. Let $D / S^{+}$ 
denote the subgraph left after the closed out-neighborhood $N^{+}[S]$ has been deleted from $D$. The vertex-neighbor-integrity of $D, V N I(D)$, then is defined as

$$
V N I(D)=\min _{S \subseteq V(D)}\left\{|S|+m\left(D / S^{+}\right)\right\}
$$

And the directed vertex-neighbor-integrity, $\overline{V N I}(G)$, of a graph $G$ is defined as $\overline{V N I}(G)=\max _{D}\{V N I(D): D$ is an orientation of $G\}$.

A set $S \subseteq V(D)$ is called an optimal subset of $V$ if $V N I(D)=|S|+m\left(D / S^{+}\right)$.

\section{Some Basic Properties of Vertex-Neighbor-Integrity of Digraphs}

In this section we present some basic properties of the vertex-neighbor-integrity of digraphs.

Property 1. It is easy to see that there exist digraphs $D_{1}$ and $D_{2}$ and two subdigraphs $H_{1} \subset D_{1}$ and $H_{2} \subset D_{2}$ such that $V N I\left(D_{1}\right)>V N I\left(H_{1}\right)$ and $\operatorname{VNI}\left(\mathrm{D}_{2}\right)<\operatorname{VNI}\left(\mathrm{H}_{2}\right)$.

Property 2. $V N I(D)=1$ if and only if $D$ has no directed cycle or there exists a dominating vertex in $D$.

Proof. The sufficiency is obvious. Suppose that $V N I(G)=1$ and $D$ has a directed cycle. Let $S$ be a subset of $V$ such that $V N I(D)=|S|+m\left(D / S^{+}\right)=1$. Since $D$ has a directed cycle necessarily $|S|=1$ and $m\left(D / S^{+}\right)=0$, hence $D$ has a dominating vertex.

Property 3. If $D^{*}$ is a digraph obtained from a digraph $D$ by reversing one arc of $D$, then $\operatorname{VNI}(D)-1 \leq V N I\left(D^{*}\right) \leq V N I(D)+1$.

Proof. By symmetry, it suffices to show that $V N I\left(D^{*}\right) \leq V N I(D)+1$. Suppose that $u v \in A(D)$ and $D^{*}$ is obtained from $D$ by reversing $u v$. Then $d_{D^{*}}^{+}(u)=$ $d_{D}^{+}(u)-1$ and $d_{D^{*}}^{-}(u)=d_{D}^{-}(u)+1$. Let $S$ be a subset of V such that $V N I(D)=$ $|S|+m\left(D / S^{+}\right)$. Note that $S$ may be empty. Let $S_{1}=S \cup\{v\}$. Then $m\left(D^{*} / S_{1}^{+}\right) \leq$ $m\left(D / S^{+}\right)$, consequently $V N I\left(D^{*}\right) \leq\left|S_{1}\right|+m\left(D^{*} / S_{1}^{+}\right) \leq|S|+1+m\left(D / S^{+}\right)=$ $1+V N I(D)$.

Property 4. Let $u$ be a vertex of $D$ with $d^{+}(u)=0$. If $D-u$ has no dominating vertex, then $V N I(D-u)=V N I(D)$.

Proof. If $D$ is acyclic, then too is $D-u$. In this case, since $D-u$ has no dominating vertex, we have $V N I(D-u)=V N I(D)=1$. Assume that $D$ contains a directed cycle. Since $d^{+}(u)=0, u$ is not in any directed cycle of $D$. If $S \subseteq V$ such that $V N I(D)=|S|+m\left(D / S^{+}\right)$, then $u \notin S$. For otherwise, $v \notin S$ for any $v \in N^{-}(u)$. But then $m\left(D / S_{1}^{+}\right)=m\left(D / S^{+}\right)$for $S_{1}=S-u$, and hence 
$\left|S_{1}\right|+m\left(D / S_{1}^{+}\right)=|S|-1+m\left(D / S^{+}\right)=V N I(D)-1$, a contradiction. If $S \cap$ $\{u\}^{-} \neq \emptyset$, then clearly $V N I(D-u)=V N I(D)$. Finally suppose that $S \cap\{u\}^{-}=$ $\emptyset$ for every optimal subset $S$ of $V$. Then we have $m\left((D-u) / S^{+}\right)=m\left(D / S^{+}\right)$and $V N I(D-u) \leq|S|+m\left((D-u) / S^{+}\right)=|S|+m\left(D / S^{+}\right)=V N I(D)$. Let $T$ be an optimal subset of $V(D-u)$. Then $m\left(D / T^{+}\right)=m\left((D-u) / T^{+}\right)$, as $d^{+}(u)=0$. Now $V N I(D) \leq|T|+m\left(D / T^{+}\right)=|T|+m\left((D-u) / T^{+}\right)=V N I(D-u)$. Hence $V N I(D)=V N I(D-u)$.

Remark 1. Let $u \in V(D)$. If $d^{+}(u)=0$ and $D-u$ has no dominating vertex, then no optimal subset $S$ of $V$ contains $u$.

Property 5. Let $D$ be a digraph with $\delta^{-}(D)>0$ and contain a directed cycle. Then $V N I(D)=2$ if and only if at least one of the following holds.

(i) $\quad m(D)=2$;

(ii) there exists $u$ in $D$ such that $D /\{u\}^{+}$is acyclic;

(iii) there exist distinct $u$ and $v$ in $D$ such that $N^{+}[u] \cup N^{+}[v]=V(D)$.

Proof. By the assumption $D$ has a directed cycle and has no dominating vertex. By Property 2 we have $V N I(D) \geq 2$. If one of $(i),(i i)$ and $(i i i)$ occurs, then certainly we have $V N I(D) \leq 2$ and hence $V N I(D)=2$. Suppose $V N I(D)=2$, and let $S$ be a subset of $V$ such that $V N I(D)=|S|+m\left(D / S^{+}\right)=2$. The possible values of $\left(S, m\left(D / S^{+}\right)\right)$are $(0,2),(1,1)$ and $(2,0)$, which imply that at least one of $(i),($ ii $)$ and $($ iii $)$ holds.

\section{Directed Vertex-Neighbor-Integrity of $K_{n}$ and $K_{s, t}$}

In this section, we first present bounds for the directed vertex-neighbor-integrity of $K_{n}$, and then we determine the directed vertex-neighbor-integrity of $K_{s, t}(s \leq$ t) for $s=3,4$.

We give an orientation, denoted by $D_{n}$, of a complete graph $K_{n}$. Let $V\left(D_{2}\right)=$ $\left\{v_{1}, v_{2}\right\}$ and $A\left(D_{2}\right)=\left(v_{1}, v_{2}\right)$. Suppose $D_{n}$ has been defined. Then $D_{n+1}$ is orientated according to the following regulations.

When $n+1$ is odd, $V\left(D_{n+1}\right)=V\left(D_{n}\right) \cup\left\{v_{n+1}\right\}$ and $A\left(D_{n+1}\right)=A\left(D_{n}\right) \cup$ $\left\{\left(v_{i}, v_{n+1}\right)\right.$ when $d^{-}\left(v_{i}\right) \geq \frac{n}{2}$, and $\left(v_{n+1}, v_{i}\right)$ otherwise $\}$.

When $n+1$ is even, $V\left(D_{n+1}\right)=V\left(D_{n}\right) \cup\left\{v_{n+1}\right\}$ and $A\left(D_{n+1}\right)=A\left(D_{n}\right) \cup$ $\left\{\left(v_{i}, v_{n+1}\right)\right.$ when $i \leq \frac{n+1}{2}$, and $\left(v_{n+1}, v_{i}\right)$ otherwise $\}$.

Theorem 1. Let $D_{n}(n \geq 2)$ be the orientation of $K_{n}$ defined as above. Then

$$
V N I\left(D_{n}\right)= \begin{cases}1, & n=2, \\ 2, & n \text { is odd or } n \text { is even with } 4 \leq n \leq 10 \\ 3, & n \text { is even with } n \geq 12\end{cases}
$$

Proof. Suppose $n$ is odd. By the definition of $D_{n}$, the vertex $v_{n}$ dominates the vertices $u$ with $d^{+}(u)-d^{-}(u)=1$. In $D_{n}$, as $n-1$ is even, $v_{n-1}$ dominates the vertices $v_{j}$ for $\frac{n-1}{2}+1 \leq j \leq n, j \neq n-1$, and there are $\operatorname{arcs}\left(v_{i}, v_{n-1}\right)$ for $i \leq \frac{n-1}{2}$. In $D_{n}$ the vertex $v_{n}$ dominates the vertices $v_{i}$ for $i \leq \frac{n-1}{2}$. Hence 
$N^{+}\left[v_{n-1}\right] \cup N^{+}\left[v_{n}\right]=V\left(D_{n}\right)$, and $V N I\left(D_{n}\right) \leq 2$. On the other hand, since $D_{n}$ contains directed cycles, we have $V N I\left(D_{n}\right) \geq 2$. Thus $V N I\left(D_{n}\right)=2$.

Now let $n \in\{4,6,8,10\}$. It is easy to check that $\left\{v_{1}, v_{2}\right\},\left\{v_{2}, v_{3}\right\},\left\{v_{1}, v_{4}\right\}$ and $\left\{v_{2}, v_{5}\right\}$ are dominating sets in $D_{n}$ for $n=4,6,8,10$, respectively. Hence in this case $\operatorname{VNI}\left(D_{n}\right)=2$ by Property 5 .

Let $n$ be even with $n=2 k \geq 12$. It is not difficult to see that $D_{2 k} / S^{+}=\left\{v_{k}\right\}$ for $S=\left\{v_{2 k-1}, v_{2 k}\right\}$, and consequently $V N I\left(D_{2 k}\right) \leq 3$. Since $D_{2 k}$ is strongly connected, we have $m\left(D_{2 k}\right)>2$. Hence to prove $V N \bar{I}\left(D_{2 k}\right) \geq 3$, by Property 5 , it suffices to show

(a) there is no vertex $v_{i}$ such that $D_{2 k} /\left\{v_{i}\right\}^{+}$is acyclic, and

(b) there are no two vertices $v_{i}$ and $v_{j}(i \neq j)$ in $D_{2 k}$ such that $N^{+}\left[v_{i}\right] \cup$ $N^{+}\left[v_{j}\right]=V\left(D_{2 k}\right)$.

We first show $(a)$. By the definition of $D_{2 k}, d^{+}\left(v_{i}\right)-d^{-}\left(v_{i}\right)=1$ for $v_{i} \in$ $\left\{v_{1}, \ldots, v_{k}\right\}$ and $d^{+}\left(v_{j}\right)-d^{-}\left(v_{j}\right)=1$ for $v_{j} \in\left\{v_{k+1}, \ldots, v_{2 k}\right\}$. Since all vertices in $\left\{v_{1}, \ldots, v_{k}\right\}\left(\left\{v_{k+1}, \ldots, v_{2 k}\right\}\right)$ have equal positions in $D_{2 k}$, it suffices to check $D_{2 k} /\left\{v_{1}\right\}^{+}$and $D_{2 k} /\left\{v_{2 k}\right\}^{+}$, respectively. Again by the definition of $D_{2 k}, v_{1}$ dominates $v_{2 j}$ for $j=1,2, \ldots, \frac{k}{2}$, so we have $D_{2 k} /\left\{v_{1}\right\}^{+}=\left\{v_{3}, v_{5}, \ldots, v_{2 k-1}\right\}$ and $v_{3} v_{5} v_{7} v_{3}$ is a cycle in $D_{2 k} /\left\{v_{1}\right\}^{+}$. Similarly, $v_{1} v_{2} v_{3} v_{1}$ is a cycle in $D_{2 k} /\left\{v_{2 k}\right\}^{+}$.

We prove $(b)$ by induction on $n$. When $n=12$, it is easy to see that $D_{12}$ contains no vertices $u$ and $v$ such that $N^{+}[u] \cup N^{+}[v]=V\left(D_{12}\right)$. Suppose that (b) holds for $D_{2 l}$ with $6 \leq l<k$.

Now suppose that $D_{2 k}$ contains $u$ and $v$ such that $N^{+}[u] \cup N^{+}[v]=V\left(D_{2 k}\right)$. Then $\{u, v\} \cap\left\{v_{2 k-1}, v_{2 k}\right\} \neq \emptyset$, for otherwise $N^{+}[u] \cup N^{+}[v]=V\left(D_{2 k-2}\right)$, contradicting the induction hypothesis. In $D_{2 k}$, as $N^{+}\left(v_{2 k-2}\right)=\left\{v_{k}, \ldots, v_{2 k-3}\right\}$ and $N^{+}\left(v_{2 k-1}\right)=\left\{v_{1}, v_{2} \ldots, v_{k-1}\right\}$, we have $\left\{v_{2 k-2}, v_{2 k-1}\right\} \cap\{u, v\}=\emptyset$. So we may assume $u=v_{2 k}$ and $v \in\left\{v_{1}, \ldots, v_{2 k-3}\right\}$. Since in $D_{2 k}$ the vertex $v_{2 k}$ dominates $v_{i}$ with $k+1 \leq i \leq 2 k-1$, the vertex $v$ must dominate $v_{j}$ with $1 \leq j \leq k$. But then in $D_{2 k-2}$, we would have $N^{+}(v) \cup N^{+}\left(v_{2 k-1}\right)=V\left(D_{2 n-2}\right)$, contradicting the induction hypothesis.

Hence $(b)$ is proved and this completes the proof of the theorem.

Theorem 2. If $n$ is an integer not less than 2, then $\overline{V N I}\left(K_{n}\right) \leq\left\lceil\log _{2} \frac{n}{2}\right\rceil+1$.

Proof. Suppose $D$ is a tournament with $n$ vertices. In the following, to get the upper bound we shall determine a subset $S$ of $V$ such that $|S|+m\left(D / S^{+}\right)$can not excess the upper bound.

For technical reason we denote $D_{1}=D$ and $n_{1}=n$. $D_{1}$ has a vertex $u_{1}$ such that $d_{D_{1}}^{+}\left(u_{1}\right) \geq\left\lfloor\frac{n_{1}}{2}\right\rfloor$. Set $D_{2}=D_{1} /\left\{u_{1}\right\}^{+}$and $n_{2}=n_{1}-\left|N_{D_{1}}^{+}\left[u_{1}\right\rfloor\right| \leq$ $n_{1}-\left\lfloor\frac{n_{1}}{2}\right\rfloor-1 \leq\left\lfloor\frac{n_{1}}{2}\right\rfloor$. And $D_{2}$ has a vertex $u_{2}$ such that $d_{D_{2}}^{+}\left(u_{2}\right) \geq\left\lfloor\frac{n_{2}}{2}\right\rfloor$. We continue this process until some $D_{k+1}$ has at most 2 vertices. Then by the construction, we have $n_{k+1} \leq\left\lfloor\frac{n_{k}}{2}\right\rfloor$. For simplicity, we assume that $n_{i+1} \leq \frac{n_{i}}{2}$ for $i=2, \ldots, k-1$ and $\frac{n}{2^{k}} \leq 2$. Then we have $n_{k+1} \leq \frac{n}{2^{k}} \leq 2$, consequently $k \geq$ $\log _{2} \frac{n}{2}$. The minimum value of $k$ is $\left\lceil\log _{2} \frac{n}{2}\right\rceil$. We set $S=\left\{u_{1}, u_{2}, \ldots, u_{k}\right\}$ with $k=$ $\left\lceil\log _{2} \frac{n}{2}\right\rceil$. Then $m\left(D / S^{+}\right) \leq 1$, and consequently $V N I(D) \leq|S|+m\left(D / S^{+}\right) \leq$ $\left\lceil\log _{2} \frac{n}{2}\right\rceil+1$. Having chosen $D$ arbitrarily, we have obtained the upper bound. 
Corollary 1. Let $n$ be an integer not less than 2. Then

(i) $2 \leq \overline{V N I}\left(K_{n}\right) \leq\left\lceil\log _{2} \frac{n}{2}\right\rceil+1$, if $n$ is odd or $n \in\{4,6,8,10\}$;

(ii) $3 \leq \overline{V N I}\left(K_{n}\right) \leq\left\lceil\log _{2} \frac{n}{2}\right\rceil+1$, if $n$ is even with $n \geq 12$.

Let $D$ be an orientation of $K_{s, t}(s \leq t)$ with bipartition $(X, Y)$. We label $X=$ $\left\{x_{1}, \ldots, x_{s}\right\}$ and $Y=\left\{y_{1}, \ldots, y_{t}\right\}$.

Proposition 1. $\overline{V N I}\left(K_{3, t}\right)=2$, where $t \geq 3$.

Proof. We prove the proposition by using Property 5 . Clearly $\overline{V N I}\left(K_{3, t}\right) \geq 2$. Let $D$ be an orientation of $K_{3, t}$. We consider the out-degrees of vertices in $Y$.

If $N^{+}(Y)=\bigcup_{y_{i} \in Y} N^{+}\left(y_{i}\right) \neq X$, then there exists a vertex $x_{k} \in X$ such that $N^{+}\left(x_{k}\right)=Y$, and $D /\left\{x_{k}\right\}^{+}$is acyclic. So in this case $V N I(D) \leq 2$. Next suppose $\bigcup_{y_{i} \in Y} N^{+}\left(y_{i}\right)=X$. If there exists, in addition, $y_{k} \in Y$ with $d^{+}\left(y_{k}\right) \geq 2$, then $D /\left\{y_{k}\right\}^{+}$is acyclic, and again $V N I(D) \leq 2$. If $d^{+}\left(y_{i}\right) \leq 1$ for every $y_{i} \in Y$ then, by Property 4 , we only need to consider the vertices $y_{j}$ with $d^{+}\left(y_{j}\right)=1$. And in this subcase, $D /\left\{x_{i}\right\}^{+}$is acyclic for every $x_{i} \in X$, consequently again we have $V N I(D) \leq 2$. Hence $\overline{V N I}\left(K_{3, t}\right) \leq 2$.

Proposition 2. $\overline{V N I}\left(K_{4, t}\right)=3$, where $t \geq 4$.

Proof. First we show $\overline{V N I}\left(K_{4, t}\right) \geq 3$. Let $D$ be an orientation of $K_{4, t}$ defined as follows. For a vertex $y_{i} \in Y$, we define $N^{+}\left(y_{i}\right)=\left\{x_{1}, x_{2}\right\}$ when $i=1(\bmod 4)$; $N^{+}\left(y_{i}\right)=\left\{x_{1}, x_{3}\right\}$ when $i=2(\bmod 4) ; N^{+}\left(y_{i}\right)=\left\{x_{2}, x_{4}\right\}$ when $i=3(\bmod 4)$ and $N^{+}\left(y_{i}\right)=\left\{x_{3}, x_{4}\right\}$ when $i=0(\bmod 4)$, and the remaining edges are oriented on the opposite direction. Let $S$ be a subset of $V(D)$. Since we want to prove $V N I(D) \geq 3$, we may assume that $|S|<3$. If $S$ contains only the vertices in $X$, then since $d^{+}\left(y_{i}\right)=2$ for every vertex $y_{i} \in Y$, we infer that $S$ must contain at least two vertices to make $D / S^{+}$be acyclic, so in this case $|S|+m\left(D / S^{+}\right)=3$. If $S$ contains the vertices in both $X$ and $Y$, then $S$ consists of one vertex in $X$ and one vertex in $Y$, by construction obviously $S$ is not a dominating set and $D / S^{+}$is acyclic. Thus $|S|+m\left(D / S^{+}\right)=3$. Suppose $S$ contains only the vertices in $Y$. If $|S|=1$, then obviously $|S|+m\left(D / S^{+}\right)=5$. Hence we assume $|S|=2$, say $S=\left\{y_{1}, y_{2}\right\}$, then also $|S|+m\left(D / S^{+}\right)=3$. So from all cases above, $|S|+m\left(D / S^{+}\right) \geq 3$ for any $S \subseteq V(D)$. Hence $V N I(D)=3$ and $\overline{V N I}\left(K_{4, t}\right) \geq 3$.

Next we show $\overline{V N I}\left(K_{4, t}\right) \leq 3$. Let $D$ be an arbitrary orientation of $\bar{K}_{s, t}$. If $N^{+}(Y)=\bigcup_{y_{i} \in Y} N^{+}\left(y_{i}\right) \neq X$, then there exists a vertex $x_{k} \in X$ such that $N^{+}\left(x_{k}\right)=Y$, and consequently $V N I(D) \leq 2$ in this case.

Now suppose $\bigcup_{y_{i} \in Y} N^{+}\left(y_{i}\right)=X$.

Case 1. There exists $y_{k} \in Y$ with $d^{+}\left(y_{k}\right) \geq 3$.

In this case $m\left(D / S^{+}\right)=1$ for $S=\left\{y_{k}\right\}$, hence $V N I(D) \leq|S|+m\left(D / S^{+}\right) \leq 2$. 
Case 2. There exists a vertex $y_{k} \in Y$ such that $d^{+}\left(y_{k}\right)=\max _{y_{i} \in Y} d^{+}\left(y_{i}\right)=2$.

If there exists a vertex $x_{l} \in X$ with $d^{-}\left(x_{l}\right)=1$ then $D /\left\{x_{l}\right\}^{+}$is acyclic and $V N I(D) \leq\left|\left\{x_{l}\right\}\right|+m\left(D /\left\{x_{l}\right\}^{+}\right)=2$. In the remaining subcase we choose an $x_{j} \in X-N^{+}\left(y_{k}\right)$, and set $S=\left\{y_{k}, x_{j}\right\}$. Then $m\left(D / S^{+}\right)=1$, and again $V N I(D) \leq 3$.

Case 3. $d^{+}\left(y_{i}\right) \leq 1$ for every $y_{i} \in Y$.

Setting $S=\left\{x_{j}\right\}$ for arbitrary $x_{j} \in X$, we have $m\left(D / S^{+}\right)=1$ since all vertices $y_{i}$ in $D / S^{+}$have no out-neighbors to $X$. Hence $V N I(D) \leq|S|+m\left(D / S^{+}\right)=2$.

From all above we have $\overline{V N I}\left(K_{4, t}\right) \leq 3$, and hence $\overline{V N I}\left(K_{4, t}\right)=3$.

\section{Acknowledgements}

We would like to thank Professor R. Vandell for sending us his papers [9] and [10], which motivate the presentation of this paper.

\section{References}

1. Barefoot, C.A., Entringer. R., Swart, H.: Vulnersbility in graphs - a comparative survey, J. Combin. Math. Combin. Comp. 1 (1987) 13-22.

2. Barefoot, C.A., Entringer. R., Swart, H.: Integrity of trees and powers of cycles, Congr. Numer. 58 (1987) 103-114.

3. Cozzens, M.B., Wu, S.-S.Y.: Vertex-neighbor-integrity of trees, Ars. Combin. 43 (1996) 169-180.

4. Cozzens, M.B., Wu, S.-S.Y.: Vertex-neighbor-integrity of powers of cycles, Ars. Combin. 48 (1998) 257-270.

5. Cozzens, M.B., Wu, S.-S.Y.: Relations between vertex-neighbor-integrity and other parameters, Ars. Combin. 55 (2000) 271-282.

6. Cozzens, M.B., Wu, S.-S.Y.: Edge-neighbor-integrity of trees, Australas. J. Combin.10 (1994) 163-174.

7. Cozzens, M.B., Wu, S.-S.Y.: Bounds for edge-neighbor-integrity of graphs, Australas. J. Combin.15 (1997) 71-80.

8. Gambrell, M.J.: Vertex-neighbor-integrity of magnifiers, expanders, and hypercubes, Discrete Math. 216 (2000) 257-266.

9. Goddard, W.G., Vandell, R.C.: The integrity of a directed graph and the directed integrity of a graph, to appear.

10. Vandell, R.C.: Maximum arc-integrity of tournaments and bipartite tourments, to appear in : Proceedings of the ninth quadrennial international conference in graph theory, combinatorics, algorithm, and applications.

11. Wu. S.-S.Y., Cozzens, M.B.: The minimun size of critically m-neighbor-connected graphs, Ars. Combin. 29 (1990) 149-160. 